\title{
Calpain and the glutamatergic synapse
}

\author{
Shachee Doshi ${ }^{1}$, David R. Lynch ${ }^{1,2}$ \\ ${ }^{I}$ Division of Neurology, Children's Hospital of Philadelphia, Philadelphia, Pennsylvania, ${ }^{2}$ Department of Neurology, University \\ of Pennsylvania, Philadelphia, Pennsylvania
}

\section{TABLE OF CONTENTS}

1. Abstract

2. Introduction

3. Calpain - a major neuronal protease

4. Glutamate receptors and calpain

4.1. The NMDA receptor and calpain

4.1.1. The NMDA receptor as a calpain substrate

4.1.2. Control of calpain-mediated cleavage of NMDARs: MAGUKS and tyrosine kinases

4.2. The AMPA receptor and calpain

4.2.1. Control of calpain-mediated cleavage of AMPARs by kinases

5. Conclusion

4.3. The metabotropic glutamate receptor and calpain

6. Acknowledgements
7. References

\section{ABSTRACT}

Calpain is a ubiquitous protease found in different tissue types and in many organisms including mammals. It generally does not destroy its large variety of substrates, but more commonly disrupts their function. In neurons, many of its substrates become dysregulated as a result of cleavage of their regulatory domain by this protease, leading to altered signaling between cells. In glutamatergic synaptic transmission, direct targets of calpain include all of the major glutamate receptors: NMDA receptors, AMPA receptors and mGluR. By cleaving these receptors and associated intracellular proteins, calpain may regulate the physiology at glutamatergic synapses. As a result, calpain-mediated cleavage in neurons might not only be involved in pathological events like excitotoxicity, but may also have neuroprotective effects and roles in physiological synaptic transmission.

\section{INTRODUCTION: GLUTAMATERGIC TRANSMISSION}

Most excitatory synapses in the mammalian nervous system utilize the amino acid neurotransmitter glutamate. Glutamate receptors are either ionotropic (those that permit ion specific channels to open after agonist stimulation) or metabotropic (those that activate downstream intracellular pathways by coupling to GTP binding proteins) $(1,2,3)$. The ionotropic glutamate receptors are further divided into three pharmacological types based on their prototypic agonist: the N-methyl-Daspartate (NMDA), the alpha-amino-3-hydroxy-5methylisoxazole-4-propionate (AMPA) and the kainate receptors, all possessing an intrinsic ion channel. The majority of these ionotropic receptors are located postsynaptically, although the NMDA receptor has been found pre- and extrasynaptically as well. Metabotropic glutamate receptors (mGluRs) are located pre-, post-, and 


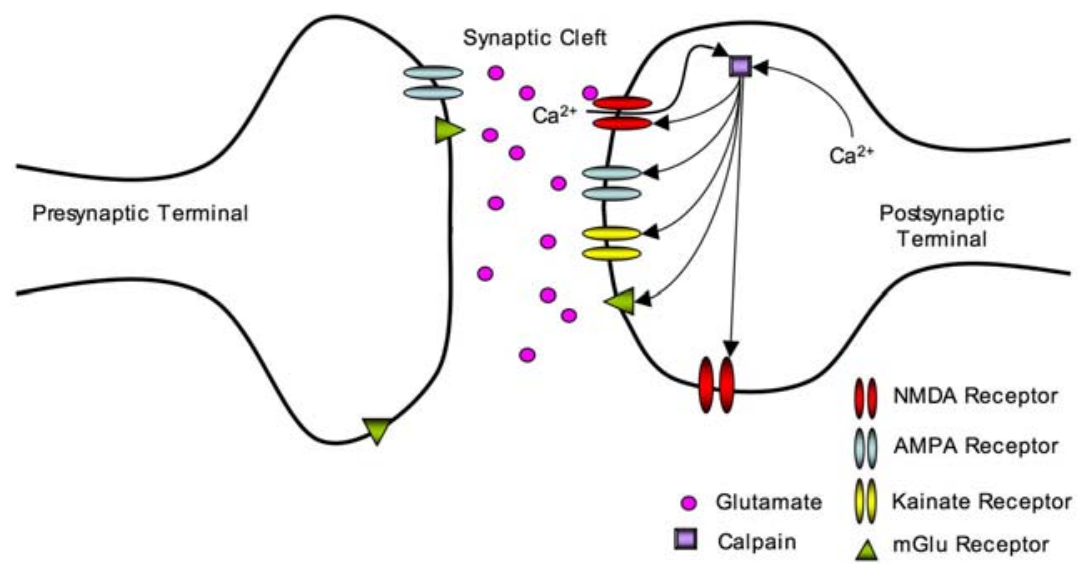

Figure 1. A glutamatergic synapse showing the major glutamate receptors. NMDARs are mostly located on the postsynaptic end of the synapse and are present at both synaptic and extrasynaptic locations, while AMPARs and mGluRs are located both preand postsynpatically. Calcium entry through synaptic NMDARs leads to activation of the protease calpain downstream of the receptor. When activated, calpain forms part of a feedback loop for controlling excessive calcium influx by cleaving most glutamate receptors and leading to a reduction in their function.

extrasynaptically, and fall into three categories based on their amino acid sequences and pharmacological sensitivities. All of these receptors are of crucial importance in excitatory signaling pathways, memory formation and long-term plasticity $(4,5)$. (Figure 1)

\section{CALPAIN - A MAJOR NEURONAL PROTEASE}

One of the downstream targets of glutamatergic transmission is calpain. Calpain is a neutral, calcium activated, intracellular cysteine protease expressed both in the cytosol and the synaptic terminal in neurons $(6,7,8,9)$. It is vital to mammalian cellular functions as well as development; disruption of the obligatory regulatory subunit of calpain in genetically engineered mice is lethal at embryonic day 11.5 (10). Calpain-mediated proteolysis in the central nervous system is not only an important event in synaptic transmission but also in neuronal death associated with pathologic processes including brain injury, ischemia and neurodegenerative disorders like Alzheimer's disease, Huntington's disease and Prion-related encephalophathy $(11,12,13,14,15)$. In the nervous system, calpain is activated by calcium influx through surface receptors or release of calcium from intracellular stores, such as the endoplasmic reticulum $(16,17,18)$. In pathologic situations, however, it can also be activated by other mechanisms including calcium influx due to nitric oxide-mediated excitotoxicity (19).

There are at least fifteen different types of mammalian calpains. Two forms of calpain are ubiquitously present in the brain: $\mu$-calpain, composed of the catalytic subunit calpain 1 in combination with the regulatory subunit calpain 4 , achieves half-maximal activity in vitro at micromolar levels of calcium $(2-80 \mu \mathrm{M})$, while m-calpain, composed of the catalytic subunit calpain
2 along with calpain 4 , requires higher levels of calcium in vitro $(0.2-0.8 \mathrm{mM})$. The other forms of calpain (calpains 3 , $5-15)$ are frequently tissue specific and less well understood $(20,21)$. Both regulatory and structural proteins are substrates for calpain $(22,12)$. Biochemically, most calpains cleave similar substrates in vitro including cytoskeletal proteins such as spectrin, tau, microtubule associated proteins like MAP2 and MAP1B, glial fibrillary acidic protein (GFAP), tubulin and neurofilaments. In neurons, other calpain substrates include receptors on the cell membrane, proteins in the postsynaptic density and intracellular proteins that contribute to synaptic signaling and structure $(12,18,23)$.

Conservation of the basic structural regions between the two ubiquitous calpains may account for the similarity in their substrate specificity. Both utilize a 30 $\mathrm{kDa}$ regulatory subunit (calpain 4) containing two domains, $\mathrm{V}$ and VI, of which domain VI is the calcium-binding domain. The larger subunit of calpain is the $80 \mathrm{kDa}$ catalytic subunit, which contains four domains (I - IV). Domain IV is also a calcium-binding domain. Calpain is also a substrate for itself, with the autolytic cleavage site located on domain I of the large subunit (2).

Calpastatin is an endogenous protein that selectively inhibits both calpain forms. It interacts with domain II of the $80 \mathrm{kDa}$ subunit (the substrate binding region), thus preventing substrate binding in a competitive manner. Calpastatin acts as an important calpain modulator and functions in a use-dependent manner; it binds to calpain only with increases in intracellular calcium (18, 25). However, the duration of action of calpastatin is limited because it is itself a substrate for calpain. New commercially produced calpain inhibitors are derived from the calpastatin amino acid sequence (26). 
Since various synaptic glutamate receptors are substrates for calpain (Figure 1), investigating the cleavage of these proteins can illuminate the role of calpain in modulating glutamatergic synaptic transmission in physiological and pathological conditions. This article explores some of the role of calpain-mediated cleavage in controlling glutamatergic signaling.

\section{GLUTAMATE RECEPTORS AND CALPAIN}

\section{1. The NMDA receptor and calpain}

The NMDA receptor (NMDAR) is an ionotropic glutamate receptor involved in models of learning and memory such as long-term potentiation and long-term depression $(1,27,28)$. The NMDAR has also been implicated in various neurological disorders such as ischemia, epilepsy and stroke, as well as many neurodegenerative diseases $(13,18,14)$. Activation of NMDARs requires simultaneous binding of two agonists (glutamate and glycine) along with membrane depolarization. The latter is required to remove a voltagedependent channel blockade by magnesium that prevents ion passage. Many other endogenous and synthetic agents also modulate the NMDAR. The receptor exists as a heterotetrameric channel in which both its major subunits (NR1 and NR2) must be usually present in order to form a functional NMDAR. It is composed of two NR1 subunits and two NR2 subunits. The NR1 subunit, which contains the glycine-binding site, is expressed as eight different splice variants NR1a-h encoded by a single gene. Four distinct genes encode the four NR2 subunit subtypes NR2A-D, which contain the glutamate-binding site (29, 18). Through its interactions with various molecules like protein kinases, calmodulin, yotiao, and alpha-actinin, NR1 can regulate receptor localization and downstream signaling (30). The NR2 subunit interacts with anchoring proteins like postsynaptic density-95 (PSD-95), Chapsyn 110/PSD-93, synapse associated protein 102 (SAP 102), and SAP $97(30,31)$. The NR2 subunit subtype(s) present in the receptor determine most NMDAR properties. NR2B levels are highest earlier in development, especially in hippocampal and cortical neurons, while NR2A levels are lower and increase with development (32). NR2C is largely confined to the cerebellum in adult rodents and humans while NR2D levels are high early in development but concentrated in a few specific nuclei and cell types in adults $(33,34,35)$.

A third NMDAR subunit NR3 has also been identified, but relatively little is known about it so far. Two different NR3 subunits NR3A and NR3B have been distinguished to date $(36,37)$. When assembled with NR1, the NR1/NR3 receptor has a glycine binding domain, but does not bind glutamate or NMDA. NR1/NR3 receptors are activated by glycine alone, leading to a burst of firing in rat cerebrocortical neurons treated with glycine. These receptors, unlike NR1/NR2 receptors, are impermeable to calcium and are not blocked by $\mathrm{Mg}^{2+}$ (38). Additionally, when co-expressed with NR1/NR2 receptors, the NR3 subunit reduces NMDAR currents and calcium permeability (39). NR3 levels increase in the rodent CNS with development until P14, after which they decline, and are lower in the adult CNS than at birth. Fewer studies have been done in humans, but NR3 expression appears to be similar developmentally between rodents and humans (40). Spatially, it is expressed in many parts of the embryonic brain, but in the adult human brain is restricted to the cerebral cortex. NR3 subunits are minimally present in the spinal cord (39). Thus, it seems as though this subunit might play an important role in development, but a lesser role in adult brain. In contrast to NR1/NR2 containing receptors, calcium-impermeant NR3 containing receptors are unlikely to act as sources of calcium for calpain activation.

\subsubsection{The NMDAR as a calpain substrate}

When activated, the NMDAR allows calcium into the cell, leading to a variety of downstream events including calpain activation. The NMDAR has been established as a physiological calpain substrate through many lines of investigation, including work in vitro, in cell culture systems and in animal models (41). In vitro, the NR1 subunit of the NMDAR is not a substrate for calpain, but three NR2 subunits (NR2A-C) are readily cleaved by calpain at their C-terminal regions; the rate of proteolysis is similar for these three NR2 subunits. Calpain cleaves NR2A before amino acids 1279 and 1330. After proteolysis, the N-terminal fragment of the NR2A subunit remains capable of forming a functional receptor when combined with NR1a, as measured by electrophysiological properties of the channel and the ability to bind the channel antagonist MK-801. Similarly, the N-terminal fragments in calpain-cleaved NR2B and NR2C subunits should be capable of assembling with NR1 to create active NMDAR, but the exact sites of cleavage in these subunits have not been identified (41).

In situ experiments in transfected cells have shown results similar to in vitro experiments. When calpain is activated by NMDAR stimulation in transfected HEK 293 or 293 t cells, NR2A and NR2B are calpain substrates while NR1 is not. The site of NR2A cleavage in situ occurs in the region after amino acid 1051, consistent with in vitro data. In contrast with in vitro experiments, however, the Nterminal fragment of the cleaved subunit is not stable on the cell surface in situ, suggesting that other cellular pathways further degrade the calpain-generated $\mathrm{N}$-terminal fragment of NR2A. The degradation of the N-terminal product by calpain and subsequent pathways leads to a loss of MK-801 binding and a reduced number of functional NMDAR (42). HEK cells transfected with NMDARs undergo cell death following receptor activation that models many properties of excitotoxicity in neurons. Consequently, calpainmediated cleavage of NR2A in transfected cells is a protective mechanism. In situ experiments in HEK cells show that blocking calpain with calpastatin accelerates NMDAR-mediated cell death; thus, calpain activation and subsequent proteolysis of NR2A provides a mechanism for controlling excessive calcium influx into the cell through the NMDAR and the resulting excitotoxic cell death. This demonstrates one paradigm in which calpain plays an important role in NMDAR function and stability and provides a crucial mechanism for cell survival via its modulatory role on receptor levels (42). 
Results from neuronal cultures are more complex than those from transfected cells or in vitro experiments. In primary rat hippocampal cultures, in which both NR2A and NR2B subunits are present, calpain is activated after NMDAR stimulation and leads to differing levels of NR2A and NR2B cleavage. NR1 and actin are not cleaved. Interestingly, in most paradigms NR2B is the major substrate for calpain, and NR2A is degraded to a much lesser degree. Cleavage occurs at the C-terminal end of NR2B and leads to the formation of a stable breakdown product of approximately $115 \mathrm{kDa}$. Levels of this product increase with increased length of agonist exposure and coincide with the decrease in full-length NR2B levels (43). The C-terminal fragment of NR2B produced in neurons essentially matches the smallest breakdown product (roughly 1030 amino acids) generated by in vitro treatment of recombinant NR $1 / 2 B$ receptors, suggesting that the sites of calpain-mediated cleavage in neurons match those in vitro. While surface levels of full-length NR2B decrease, the cleaved NR2B remains on the surface in hippocampal neurons and is potentially active. The $115 \mathrm{kDa}$ NR2B breakdown product observed in cultured neurons after agonist treatment is also observed after transient ischemia in the hippocampus and in animals undergoing status epilepticus $(43,44)$. This indicates that calpain cleaves NMDAR subunits during excitotoxic insults in the brain as well as in cultured neurons. Even though NR2A is not significantly cleaved by calpain in more developed hippocampal neurons in culture, it is cleaved at earlier developmental time points and may be crucial in the activation of calpain in hippocampal neurons (43). This last result matches findings from HEK cells. In cells transfected with NR1A/2B, the NR2B subunit is not readily cleaved and calpain activation is minimal. However, when NR2A is also included, NR2B is cleaved by calpain (43).

Experiments in neurons derived from other brain regions produce similar results. In acutely isolated and cultured cortical neurons, calpain selectively cleaves NR2A and NR2B after NMDAR activation (11), and in vitro experiments in synaptic membranes of the rat telencephalon also demonstrate calpain-induced cleavage of the NR2 subunit (45). However, as in hippocampal cultures, such results vary developmentally. NR2A is cleaved in hippocampal and cortical neurons early in development, but cleavage levels decrease with development (DIV14-21) in both types of cells; this may reflect an increasing association of NR2A subunits with PSD-95 (see below). However, NR2B degradation by calpain remains the same in hippocampal cells over this period, while in cortical cells, it is cleaved to a lesser degree at DIV21 as compared to DIV14. Thus the cleavage of NR2 subunits by calpain appears to reflect both cell type and developmental stage.

Electrophysiological properties of the NMDAR after calpain activation also vary over development in hippocampal cultures. Agonist treatment with glutamate and glycine for 30 minutes decreases NMDAR current density in DIV21 cells but not in DIV10 cells. IN DIV21 neurons, this effect is similar in the presence or absence of calpain inhibitors; thus, calpain does not seem to control this phenomenon. This may reflect agonists induced internalization, in which binding of agonists to the NMDAR, in particular glycine, leads to receptor internalization and hence reduced current density through the channel (46). In contrast, a calpain dependent change in desensitization of NMDARs appears in DIV10 neurons after glutamate and glycine treatment. However, calpainmediated cleavage of NR2B decreases the levels of full length NR2B in the DIV10 cells, but the cleaved receptors remain on the surface and appear to remain active (47). Thus, at this time in development calpain not only readily cleaves receptors but creates a novel form of active receptor with altered desensitzation. This shows that the physiological effect of calpain-mediated NMDAR cleavage may differ over development.

A second aspect of the NMDAR-calpain interaction is the regulation of calpain activation by NMDAR subtypes. NMDAR-mediated calpain activity increases with development (DIV7 to DIV21) in hippocampal as well as cortical cultures. In hippocampal neurons, calpain activity plateaus at DIV14, but continues to increase in cortical neurons until DIV21. This increase reflects an increase in expression of NMDA subunits and is not due to increases in calpain levels. Levels of calpain I remain constant throughout the DIV7-21 period, while levels of NR1, 2A and 2B increase significantly in cortical cultures; NR1 and 2A levels increase but NR2B levels fall with development in hippocampal cultures. The NMDAR subtype associated with calpain activation is also controlled by developmental changes in subunit compositions and neuronal types. It is primarily activated by NR $1 / 2 \mathrm{~B}$ and NR $1 / 2 \mathrm{~A} / 2 \mathrm{~B}$ in DIV7 cortical cultures. At DIV14, these receptors continue to play an important role in calpain activation, but it is lower than DIV7. In contrast, NR $1 / 2 \mathrm{~B}$ is the primary receptor type involved in activation of calpain at DIV7 in hippocampal cultures; by DIV14, NR1/2A/2B also plays a significant role in this process. With further development, at DIV21, NR1/2B plays a negligible role in calpain activation while NR1/2A/2B and likely even NR1/2A contribute the most to this physiological event (47). Increased calpain activation in NR1/2A receptors could reflect the high fractional $\mathrm{Ca}^{2+}$ passage through them (48). As C-terminally truncated NR2A constructs activate calpain equally to wild-type, there is no evidence to suggest that the high level of calpain activation reflects specific structural motifs in the intracellular region of NR2A. All of these findings illustrate the changes in the role of calpain in modulating NMDAR function with development.

\subsubsection{Control of calpain-mediated cleavage of NMDARs: MAGUKS and tyrosine kinases \\ The postsynaptic density is rich in many} anchoring proteins, one of which is postsynaptic density 95 (PSD-95). The N-terminal end of PSD-95 contains a domain of 90 amino acids called PSD-95/Discs large/zone occludens-1 (PDZ) through which it binds the C-terminus of NR2 subunits (22). PSD-95 also binds many other proteins such as the microtubule-associated protein CRIPT, potassium channels, and Ras GTP-ase. This ability to bind many molecules along with its ability to form polymers facilitates NMDAR clustering and stabilization at the synapse (22). 


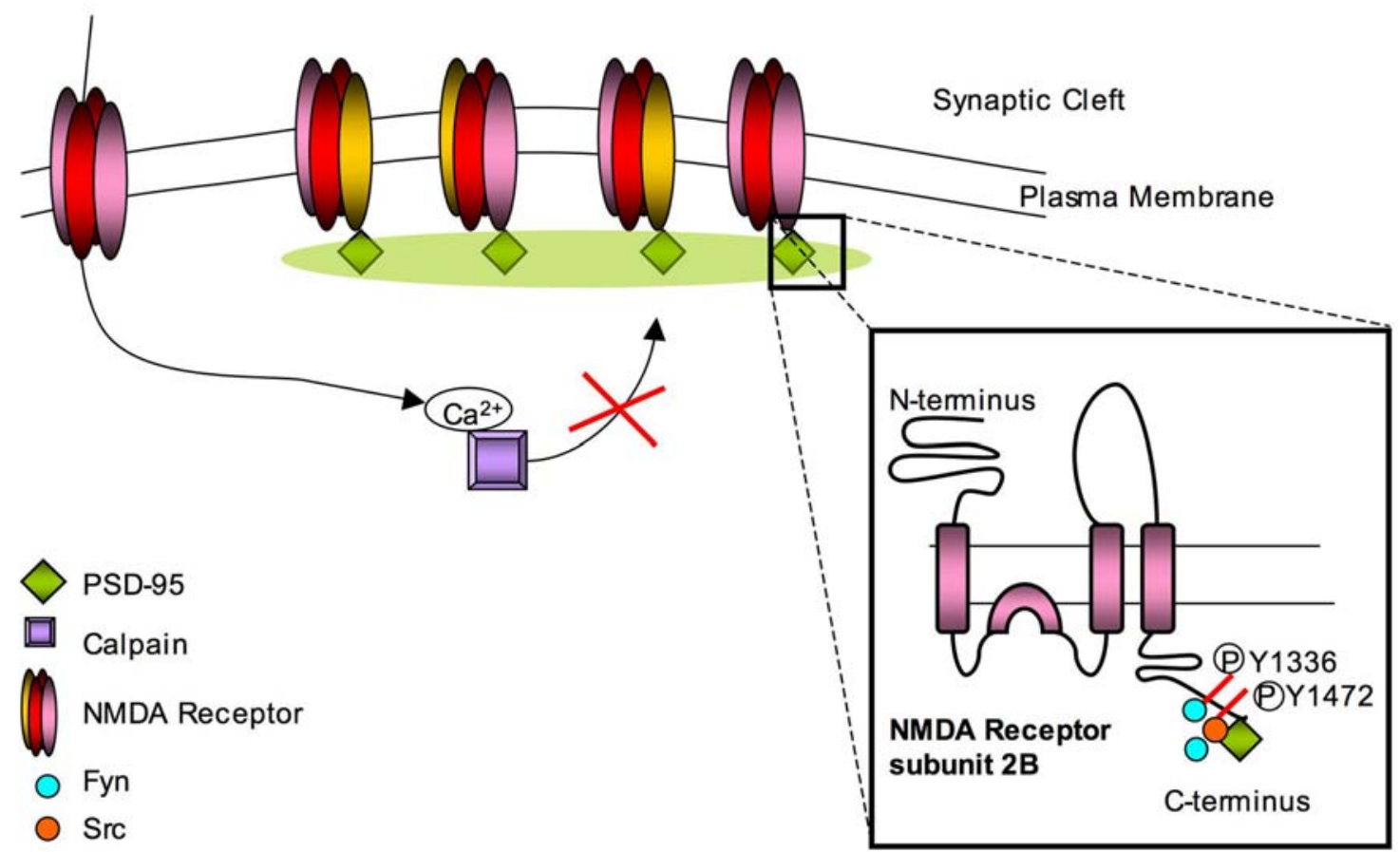

Figure 2. Role of PSD-95 and SFKs in calpain-mediated cleavage of NMDARs. PSD-95 is one of the postsynaptic density membrane associated guanylate kinases (MAGUKs) involved in clustering of NMDARs on the cell surface. It binds the Cterminus of the receptor at the ESDV motif, and protects against calpain-mediated cleavage of that end. The inset shows two Src family kinases (SFKs), Src and Fyn, that phosphorylate residues on the C-terminus of NR2B leading to differing regulation by calpain. Although the substrate specificity of Fyn and Src is similar in many situations, the inset proposes a mechanism by which they could differentially regulate calpain-mediated cleavage. Fyn could preferentially phosphorylate Y1252 and Y1336 and facilitate calpain-mediated cleavage through phosphorylation of the latter, while Src could preferentially phosphorylate Y1472 and lead to binding to PSD-95, thus preventing cleavage by calpain.

Binding of PSD-95 modulates the ability of calpain to cleave NMDARs. In HEK293 cells transfected with NMDAR subunits, PSD-95 protects NR2A from calpain cleavage both at the cell surface as well as intracellularly. NR2A binds to PSD-95 through the final four amino acids of NR2A, known as the ESDV motif (49). These amino acids are also necessary for the effect of PSD95 on calpain-mediated cleavage of NR2A. The process by which PSD-95 limits cleavage by calpain is not entirely clear, but PSD-95 does not reduce calpain activity or NMDAR- mediated intracellular calcium responses after agonist exposure, suggesting that the effect of PSD-95 is not an effect on calpain activation but instead on targeting of calpain to the NMDAR (8). It also does not require receptor internalization, since preventing internalization of NMDARs does not affect receptor cleavage. However, palmitoylation of PSD-95 is required for it to block the cleavage of NR2 by calpain. Palmitoylation sites on PSD95 allow it to cluster and stably bind the C-terminus of NR2A. SAP102, which, like PSD-95 is a membraneassociated guanylate kinase (MAGUK), is not palmitoylated, does not cluster, and does not block cleavage of NR2A by calpain. This suggests that clustering is an essential component to the effect of PSD-95 on NMDAR cleavage, and that the effect of PSD-95 may be mediated by steric hindrance (8). Regardless of the exact mechanism, the effect of PSD-95 on calpain-mediated cleavage may explain subunit selectivity of calpain for NR2B in hippocampal neurons. In these neurons, PSD-95 is associated with NR2A more than with NR2B, which makes the $2 \mathrm{~A}$ subunit resistant to calpain-mediated cleavage. In contrast, NR2B, which is found at higher levels extrasynaptically, is more readily proteolyzed. In situ studies in HEK 293 cells have shown that PSD-95 protects NR2B from calpain-mediated proteolysis, suggesting that in neurons, calpain targets predominantly extrasynaptic, rather than synaptic, NMDARs (50). (Figure 2)

A second regulatory mechanism for calpainmediated cleavage is phosphorylation of tyrosine residues. Src family kinases (SFKs) are tyrosine kinases highly expressed in neurons. Two such kinases, Src and Fyn, have been extensively studied due to their interaction with NMDA as well as AMPA receptors. Specific interactions between SFKs, MAGUK proteins, and NMDAR activation control calpain-mediated proteolysis of the NR2 subunit (Figure 2). Src is directed to NR2A and 2B subunits by selectively and uniquely binding PSD-95, among MAGUKs, to gain access to the receptors. Src-mediated phosphorylation of NMDAR subunits significantly reduces calpain-mediated cleavage of these receptors in vitro (51, 52). In contrast, Fyn-mediated phosphorylation of NR2A and NR2B enhances cleavage by calpain. Fyn phosphorylates NR2B at three distinct sites: Y1252, Y1336 
and Y1472. Of these, phosphorylation of Y1336 facilitates calpain-mediated cleavage of NR2B. However, while MAGUK proteins like PSD-95 or SAP102 are required for phosphorylation of NR2B by constitutively active Fyn, PSD-95 blocks cleavage of NR2B such that only NR2B associated with SAP102 is readily cleaved by calpain. Although Fyn and calpain are active intracellularly, and SAP102 and NR2B associate intracellularly, the Fynmediated increase of NR2B cleavage by calpain is limited to cell surface receptors (53). These data suggest that the control of NR2B cleavage involves not only MAGUK proteins and SFKs, but also other macromolecular or lipid components that vary between cellular compartments.

Interestingly, NR2B-Y1336 is involved in a variety of processes downstream from NMDAR activation. These include dephosphorylation/inactivation of the MAP kinase p38, activation of PI3 kinase (a protective mechanism in excitotoxicity), ubiquitination of NR2B, and ethanol sensitivity of the NMDAR $(54,55,56,57)$. In contrast, phosphorylation of Y1472 is linked with increased association of NR2B with PSD95 and regulation of NMDAR levels on the cell surface $(58,59)$. Moreover, phosphorylation of the different residues is altered by NMDAR activation, with Y1336 phosphorylation increasing with NMDAR activation. This leads to the possibility that the control of calpain-mediated cleavage by Y1336 is part of a feedback loop in which high levels of NMDAR activation lead to a pattern of calpain activation, p38 dephosphorylation, and PI3 kinase activation. Such events could be crucial in synaptic remodeling as well as components of excitotoxicity.

\subsection{The AMPA receptor and calpain}

AMPA receptors are the major ionotropic glutamate receptors mediating fast excitatory signaling at the synapse. Like the NMDAR, AMPA receptors are involved in various processes in the nervous system including synaptic plasticity, dendritic sprouting and regulation of gene expression (60). Such receptors are also cation channels activated by the binding of glutamate to two of the four binding sites on each receptor (61). Once activated, AMPA receptors allow the passage of only sodium and potassium in most situations. In receptors lacking the edited form of the GluR2 subunit, calcium can also pass (such receptors are a minority of brain AMPA receptors). The receptor is composed of four subunits GluR1, GluR2, GluR3 and GluR4, each possessing an extracellular $\mathrm{N}$-terminus, an intracellular $\mathrm{C}$-terminus and three transmembrane loops. The C-terminus interacts with many molecules including anchoring proteins such as GRIP and PICK1, and kinases that lead to its phosphorylation (62).

Calpain can modulate AMPA receptor function post-translationally by cleaving the $\mathrm{C}$-terminus of the GluR1 subunit. In this situation, calpain is usually activated by NMDAR- mediated calcium influx since AMPARmediated calcium influx is usually insufficient for calpain activation. This reflects the inability of most AMPA receptors to pass calcium and their fast desensitization. A small population of AMPARs allows calcium influx but the fractional calcium current is smaller than that of NMDAR
(48). However, in spite of the homology between different AMPA receptor subunits, not all are readily cleaved by calpain. In cortical pyramidal neurons activation of calpain by prolonged NMDA application leads to C-terminal GluR1 cleavage (putatively before amino acids N833 and R837), while GluR2 levels remain unchanged (63). If the NMDAR is stimulated briefly, the levels of GluR1 cleavage are much lower, and most AMPA receptor destruction occurs through endocytosis rather than calpainmediated degradation. With increased duration of NMDAR stimulation, calpain-mediated cleavage increases. The role of calpain as a regulatory mechanism for AMPA receptor turnover at high levels of NMDAR stimulation parallels the effect of NMDAR stimulation of calpain-mediated cleavage of NR2B. However, in contrast to NR2B, the calpain-generated fragment of GluR1 does not remain on the surface and is probably internalized. Similar results have been observed in models of hypoxia and ischemia, suggesting that cleavage of AMPAR by calpain acts as a mechanism to control excitotoxicity (63).

\subsubsection{Control of calpain-mediated cleavage of AMPARs by kinases}

In addition, while phosphorylation of AMPA receptors by various kinases modulates its susceptibility to calpain-mediated cleavage, the exact regulation of AMPAR cleavage by SFKs differs from that of NMDAR. In synaptic membranes, Src-mediated phosphorylation of AMPA receptors does not alter GluR1 cleavage, while Fyn protects GluR1 from being cleaved by calpain. Since there is only one tyrosine residue in the C-terminus of AMPAR, the two different SFKs may interact with other molecules in differentially regulating AMPAR susceptibility to cleavage by calpain $(64,52)$. Other kinases, like CaMKII, also modulate calpain-mediated cleavage of GluR1. Phosphorylation of S831 on GluR1 by CaMKII significantly increases cleavage of the subunit by calpain in cortical neurons in vitro. Additionally, inhibiting or reducing expression of CaMKII, or mutating S831 prevents the increased cleavage of the receptor observed upon agonist exposure (65). Overall, the modulation of calpainmediated cleavage of AMPAR by phosphorylation suggests that it is a carefully regulated system, although the exact role of this cleavage remains unclear. (Figure 3a)

\subsection{The mGluR and calpain}

Metabotropic glutamate receptors (mGluRs) mediate neuronal signaling by coupling to G-proteins associated with specific downstream targets. There are three major categories of mGluRs based on pharmacological differences and associated second messenger systems. Group I includes mGluR1 and 5, Group II includes mGluR2 and 3, and Group III includes mGluR4, 6, 7 and 8. All eight mGluRs are products of distinct genes, and many of them are further subdivided into different splice variants. Different mGluRs are activated to varying degrees by different prototypic agonists, are associated with different second messenger systems, and differ in their synaptic localization. Despite the large diversity of the mGluRs they are similar in their basic structure, possessing an extracellular and intracellular domain with seven transmembrane loops (66). 

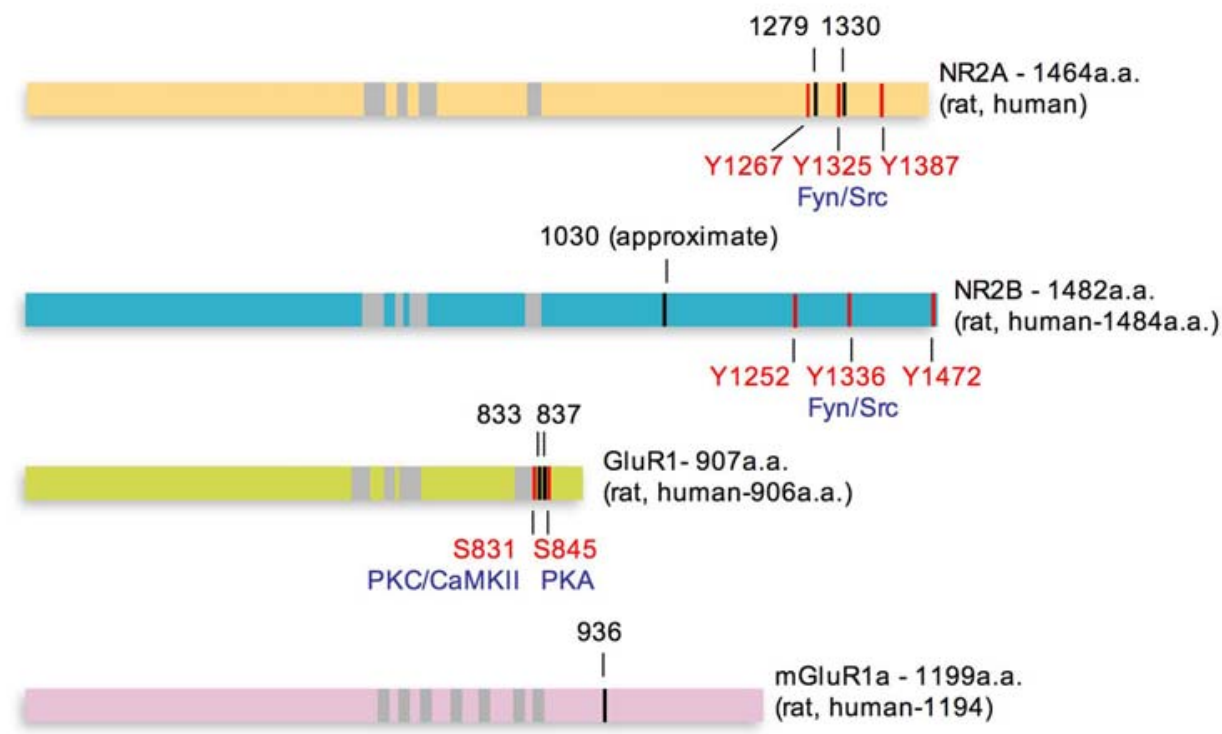

| Calpain Cleavage Site

| Phosphorylation Site

A

ITransmembrane Domain

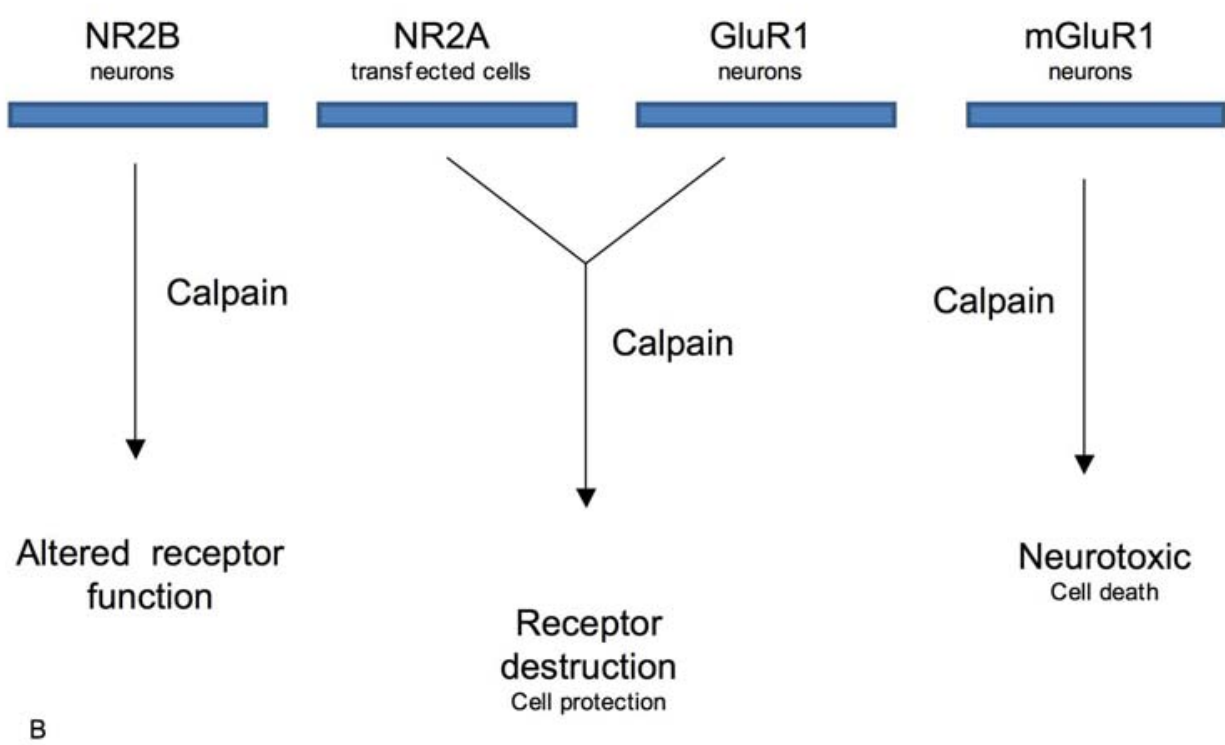

Figure 3. A.Calpain-mediated cleavage sites and selected phosphorylation sites on different subunits of the NMDA, AMPA and mGlu receptors. The locations of the transmembrane domains are highly conserved among the ionotropic receptor subunits. The exact calpain cleavage sites on NR2B are not fully known, but it should be noted that many phosphorylation sites are located proximal to calpain cleavage sites, indicating the important role played by phosphorylation of C-terminal residues in controlling calpain-mediated cleavage. B.Effect of calpain-mediated cleavage on different glutamate receptor subunits in specified cellular systems and the proposed role of calpain in the fate of the cell. 
Only recently has the mGluR family been shown to be a calpain target. mGluR1-alpha, a Group I receptor, is cleaved by calpain at its intracellular, C-terminal end at the residue S936 (66). This cleavage is induced by calcium influx through the NMDAR, and has neurotoxic effects. The cleaved mGluR1-alpha is active and remains on the cell surface, but through loss of its C-terminus, is uncoupled from downstream pathways. One such pathway is the PI3K-Akt pathway, which has neuroprotective effects that are lost upon calpain-mediated cleavage of the $\mathrm{C}$ terminus of the receptor. A fusion peptide derived from the site of mGluR1-alpha cleavage selectively blocks its cleavage and restores the neuroprotective effects of the PI3K-Akt pathway. This provides an example of a specific cellular process regulated by calpain. Thus calpainmediated cleavage of mGluR1-alpha provides a specific role for calpain in excitotoxicity as well as a link between excitotoxicity and NMDAR-mGluR1 interactions (67).

\section{CONCLUSION}

Calpain appears to be a significant regulator of synaptic transmission at the glutamatergic synapse. However, the ways in which calpain mediates cleavage of NMDARs, AMPARs and mGluRs to regulate neuronal functions remains somewhat unclear. Among glutamate receptors, calpain cleavage leads to dysregulation of their normal functions, but the exact consequences of such events in downstream signaling remain to be elucidated. (Figure 3b). However, in some physiological and pathophysiological scenarios calpain-mediated cleavage of substrates can regulate transcription. Calpain cleaves betacatenins, a group of molecules that are involved in development and onset of cancer by acting as transcriptional regulators; the calpain generated fragments of beta-catenin are stable in neurons and translocated to the nucleus where they modulate Tcf, a transcription factor (68). In another instance, calpain-mediated cleavage of specific substrates directly leads to cell death through nuclear events. The mitochondrial protein apoptosis inducing factor (AIF) is cleaved by calpain during excitotoxic events; the resulting fragment is released from the mitochondria and translocated to the nucleus during ischemic conditions. In the nucleus, AIF causes cell death and leads to genomic degradation (69).

While these specific examples of calpain dependent events aid in understanding the role of calpain, a crucial aspect that is not understood is the regulation of cleavage. The role of kinases and phosphatases in the cleavage of NMDARs by calpain has provided a greater understanding of the complex interactions of calpain with its substrates. It is plausible that such regulatory events exist for many other substrates, and a knowledge and understanding of these events would provide significant insight into the specific roles of calpain in the cell at large. Understanding of the biochemical processes involved in synaptic cleavage of glutamate receptors by calpain is thus a further goal. In cell adhesion processes, calpain mediated control of integrins is regulated by SFKs and MAPK through formation of a macromolecule complex at the cell surface (70). Conceivably, similar complexes might regulate cleavage of glutamate receptors and other synaptic substrates. However, direct demonstrations of such a mechanism are needed before we fully understand the role of calpain-mediated cleavage of glutamate receptors in neuronal function.

\section{ACKNOWLEDGEMENT}

Dr. Lynch is supported by NS45986 and by the Trisomy 21 program of the Children's Hospital of Philadelphia. The authors would like to thank Ms. Amy Gleichman for critical reading of and valuable suggestions for the manuscript.

\section{REFERENCES}

1. Graham L. Collingridge, John T. R. Isaac, Yu Tian Wang: Receptor trafficking and synaptic plasticity. Nat Rev Neurosci 5, 952-962 (2004)

2. David R. Lynch, Rodney P. Guttmann: Excitotoxicity: Perspectives based on N-methyl-D-aspartate receptor subtypes. J Pharmacol Exp Ther 3, 717-723 (2002)

3. Raymond Dingledine, Karin Borges, Derek Bowie, Stephen F. Traynelis: The glutamate receptor ion channels. Pharmacol Rev 51, 7-62 (1999)

4. Tihomir P. Obrenovitch, Jutta Urenjak: Altered glutamatergic transmission in neurological disorders: from high extracellular glutamate to excessive synaptic efficacy. Prog Neurobiol 51, 39-87 (1997)

5. Ole P. Ottersen, Alf S. Landsend: Organization of glutamate receptors at the synapse. Eur J Neurosci 9, 22192224 (1997)

6. Ronald L. Mellgren, Waleed M. Renno, Richard D. Lane: The non-lysosomal, calcium-dependent proteolytic system of mammalian cells. Revis Biol Celular 20, 139-159 (1989)

7. Edon Melloni, Sandro Pontremoli: The calpains. Trends Neurosci 12, 438-444 (1989)

8. Yi Na Dong, Elisa A. Waxman, David R. Lynch: Interactions of postsynaptic density-95 and the NMDA receptor 2 subunit control calpain-mediated cleavage of the NMDA receptor. J Neurosci 24, 11035 - 11045 (2004)

9. Yoshiro Tomimatsu, Satoru Idemoto, Shigeki Moriguchi, Shigenori Watanabe, Hiroshi Nakanishi: Proteases involved in long-term potentiation. Life Sci 72, 355-361 (2002)

10. J. Simon C. Arthur, John S. Elce, Carol Hegadorn, Karen Williams, Peter A. Greer: Disruption of the murine calpain small subunit gene, Capn4: calpain is essential for embryonic development but not for cell growth and division. Mol Cell Biol 20, 4474-4481 (2000)

11. Hai-Yan Wu, Eunice Y. Yuen, Yun-Fei Lu, Masayuki Matsushita, Hiseki Matsui, Zhen Yan, Kazuhito Tomizawa: 
Regulation of N-methyl-D-aspartate receptors by calpain in cortical neurons. J Biol Chem 280, 21588 -21593 (2005)

12. Kin Chiu, Tim Tak Lam, Winnie Wai Ying Li, Joseph Caprioli, Jacky Man Kwong Kwong: Calpain and Nmethyl-D-aspartate - induced excitotoxicity in rat retinas. Brain Res 1046, 207-215 (2005)

13. M. Flint Beal: Role of excitotoxicity in human neurological disease. Curr Opin Neurobiol 2, 657-662 (1992)

14. Anne B Young, J. Timothy Greenamyre, Zane Hollingsworth, Roger Albin, C D'Amato, Ira Shoulson, John B Penney: NMDA receptor losses in putamen from patients with Huntington's disease. Science 241, 981-983 (1988)

15. Rajgopal Yadavalli, Rodney P. Guttmann, Tanya Seward, Afrian P. Centers, R. Anthony Williamson, Glenn C. Telling: Calpain-dependent endoproteolytic cleavage of $\mathrm{PrP}^{\mathrm{Sc}}$ modulates scrapie prion propagation. $J$ Biol Chem 279, 21948-21956 (2004)

16. Hai-Yan Wu, David R. Lynch: Calpain and Synaptic Function. Mol Neurobiol 33, 215-236 (2006)

17. Ralph A. Nixon, Ken-Ichi Saito, F. Grynspan, William R. Griffin, S. Katayama, T. Honda, Panaiyur S. Mohan, Thomas B. Shea, Mary L. Beermann: Calciumactivated neutral proteinase (calpain) system in aging and Alzheimer's disease. Ann N Y Acad Sci 15, 77-91 (1994)

18. Joshua L. Hood, William H. Brooks, Thomas L. Roszman: Differential compartmentalization of the calpain/calpastatin network with the endoplasmic reticulum and golgi apparatus. J Biol Chem 279, 4312643135 (2004)

19. Christiane Volbracht, Eugenio Fava, Marcel Leist, Pierluigi Nicotera: Calpain inhibitors prevent nitric oxide-triggered excitotoxic apoptosis. Neuroreport 12, 3645-3648 (2001)

20. Matthew B. Bevers, Robert W. Neumar: Mechanistic role of calpains in postischemic neurodegeneration. J Cereb Blood Flow Metab 28, 655-673 (2008)

21. Maria E. Saez, Reposo Ramirez-Lorca, Francisco J. Moron, Agustin Ruiz: The therapeutic potential of the calpain family: new aspects. Drug Discov Today 11, 917923 (2006)

22. Lucia Vinade, Jennifer D. Peterson, Khanh Do, Ayse Dosemeci, Thomas S. Reese: Activation of calpain may alter the postsynaptic density structure and modulate anchoring of NMDA receptors. Synapse 40, 302-309 (2001)

23. Peter Tompa, Peter Buzder-Lantos, Agnes Tantos, Attila Farkas, Andras Szilagyi, Zoltan Banoczi, Ferenc Hudecz, Peter Friedrich: On the sequential determinants of calpain cleavage. J Biol Chem 279, 20775-20785 (2004)
24. Andrea Baki, Peter Tompa, Anita Alexa, Orsolya Molnar, Peter Friedrich: Autolysis parallels activation of mu-calpain. Biochem J 318, 897-901 (1996)

25. Takashi Murachi: Intracellular regulatory system involving calpain and calpastatin. Biochem Int 18, 263-294 (1989)

26. Masatoshi Maki, Huseyin Bagci, Kaoru Hamaguchi, Michiko Ueda, Takashi Murachi,

Masakazu Hatanka: Inhibition of calpain by a synthetic oligopeptide corresponding to an exon of the human calpastatin gene. J Biol Chem 264, 18866-18869 (1989)

27. Robert C. Malenka, Roger A. Nicoll: Long-term potentiation-A decade of progress? Science 285, 18701874 (1999)

28. Serena M. Dudek, Mark F. Bear: Homosynaptic longterm depression in the area CA1 of hippocampus and effects of N-methyl-D-aspartate receptor blockade. Proc Natl Acad Sci 89, 4363-4367 (1992)

29. Takahiro Ishii, Koki Moriyoshi, Hidemitsu Sugihara, Kazuhiro Sakurada, Hiroshi Kadotani, Mineto Yokoi, Chihiro Akazawa, Ryuichi Shigemoto, Noboru Mizuno, Masayuki Masu, Shigetada Nakanishi: Molecular characterization of the family of the N-methyl-D-aspartate receptor subunits. J Biol Chem 268, 2836-2843 (1993)

30. Katherine W. Roche, Steve Standley, Jennifer McCallum, C. Dune Ly, Michael D. Ehlers, Robert J. Wenthold: Molecular determinants of NMDA receptor internalization. Nat Neurosci 4, 794-802 (2001)

31. Martin Niethammer, Eunjoon Kim, Morgan Sheng: Interaction between the $\mathrm{C}$ terminus of NMDA receptor subunits and multiple members of the PSD-95 family of membrane-associated guanylate kinases. J Neurosci 16, 2157-2163 (1996).

32. Bo Li, Nansheng Chen, Tao Luo, Yo Otsu, Timothy H. Murphy, Lynn A. Raymond: Differential regulation of synaptic and extrasynaptic NMDA receptors. Nat Neurosci $5,833-834(2002)$

33. Hannah Monyer, Nail Burnashev, David J. Laurie, Bert Sakmann, Peter H. Seeburg: Developmental and regional expression in the rat brain and functional properties of four NMDA receptors. Neuron 12, 529-540 (1994)

34. Morgan Sheng, Jennifer Cummings, Leslie Ann Roldan, Yuh Nung Jan, Lily Yeh Jan: Changing subunit composition of heteromeric NMDA receptors during development of rat cortex. Nat 368, 144-147 (1994)

35. David G. Standaert, G. Bernhard Landwehrmeyer, Julie A. Kerner, John B. Penney Jr., Anne B. Young: Expression of NMDAR2D glutamate receptor subunit mRNA in neurochemically identified interneurons in the rat neostriatum, neocortex and hippocampus. Brain Res $\mathrm{Mol}$ Brain Res 42, 89-102 (1996) 
36. Nikolaus J. Sucher, Schahram Akbarian, Carlin L. Chi, Cynthia L. Leclerc, Marc Awobuluyi, David L. Deitcher, Michele K. Wu, Joseph P. Yuan, Edward G. Jones, Stuart A. Lipton: Developmental and regional expression pattern of a novel NMDA receptor-link subunit (NMDAR-L) in the rodent brain. $J$ Neurosci 15, 6509-6520 (1995)

37. Olof Andersson, Anna Stenqvist, Anneli Attersand, Gabriel von Euler: Nucleotide sequence, genomic organization and chromosomal localization of genes encoding the human NMDA receptor subunits NR3A and NR3B. Genomics 78, 178-184 (2001)

38. Jon E. Chatterton, Marc Awobuluyi, Louis S. Premkumar, Hiroto Takahashi, Maria Talantova, Yeonshook Shin, Jiankun Cui, Shichun Tu, Kevin A. Sevarino, Nobuki Nakanishi, Gang Tong, Stuart A. Lipton, Dongxian Zhang: Excitatory glycine receptors containing the NR3 family of NMDA receptor subunits. Nat 415, 793798 (2002)

39. Akira Wada, Hiroto Takahashi, Stuart A. Lipton, H.-S. Vincent Chen: NR3A modulates the outer vestibule of the "NMDA" receptor channel. J Neurosci 26, 13156-13166 (2006)

40. A. Nilsson, J. Duan, L.-L. Mo-Boquist, E. Benedikz, E. Sundström: Characterisation of the human NMDA receptor subunit NR3A glycine binding site. Neuropharmacol 52, 1151-1159 (2006)

41. Rodney P. Guttmann, Dana L. Baker, Kristen M. Seifert, Akiva S. Cohen, Douglas A. Coulter, David R. Lynch: Specific proteolysis of the NR2 subunit at multiple sites by calpain. $J$ Neurochem 78, 1083-1093 (2001)

42. Rodney P. Guttmann, Set Sokol, Dana L. Baker, Kelly L. Simpkins, Yina Dong, David R. Lynch: Proteolysis of the N-methyl-D-aspartate receptor by calpain in situ. J Pharmacol 302: 1023-1030 (2002)

43. Kelly L. Simpkins, Rodney P. Guttmann, Yina Dong, Zhaoming Chen, Set Sokol, Robert W. Neumar, David R. Lynch: Selective activation induced cleavage of the NR2B subunit by calpain. J Neurosci 23, 11322-11331 (2003)

44. Ines M. Araujo, Sara Xapelli, Joana M. A. C. Gill, Paul Mohapel, Asa Petersen, Paulo S. Pinheiro, Joao O. Malva, Ben A. Bahr, Patrik Brundin, Caetana M. Carvalho: Proteolysis of NR2B by calpain in the hippocampus of epileptic rats. Neuroreport 16, 393-396 (2005)

45. Xiaoning Bi, Yongqi Rong, Jing Chen, Sandeep Dang, Zhuo Wang, Michel Baudry: Calpain-mediated regulation of NMDA receptor structure and function. Brain Res 790, 245-253 (1998)

46. Yi Nong, Yue-Qiao Huang, William Ju, Lorraine V. Kalia, Gholamreza Ahmadian, Yu Tian Wang, Michael
W. Salter: Glycine binding primes NMDA receptor internalization. Nature 422, 302-307 (2003)

47. Yi Na Dong, Hai-Yan Wu, Fu-Chun Hsu, Douglas A. Coulter, David R. Lynch: Developmental and cell-selective variations in N-methyl-D-aspartate receptor degradation by calpain. J Neurochem 99, 206-217 (2006)

48. Nail Burnashev, Z Zhou, Erwin Neher, Bert Sakmann: Fractional calcium currents through recombinant GluR channels of the NMDA, AMPA and kainate receptor subtypes. J Physiol 485, 403-418 (1995)

49. Hans-Christian Kornau, Leslie T. Schenker, Mary B. Kennedy, Peter H. Seeburg: Domain interaction between NMDA receptor subunits and the postsynaptic density protein PSD-95. Science 269, 1737-1740 (1995)

50. Nathalie Sans, Ronald S. Petralia, Ya-Xian Wang, Jaroslav Blahos II, Johannes W. Hell, Robert J. Wenthold: A developmental change in NMDA receptor-associated proteins in hippocampal synapses. $J$ Neurosci 20, 1260$1271(2000)$

51. Ruifen Bi, Yongqi Rong, Anne Bernard, Michel Khrestchatisky, Michel Baudry: Src-mediated tyrosine phosphorylation of NR2 subunits of N-Methyl-D-Aspartate receptors protects from calpain-mediated truncation of their C-terminal domains. J Biol Chem 275, $26477-26483$ (2000)

52. Yongqi Rong, Ziaoying Lu, Anne Bernard, Michel Khrestchatisky, Michel Baudry: Tyrosine phosphorylation of ionotropic glutamate receptors by $\mathrm{Fyn}$ or $\mathrm{Src}$ differentially modulates their susceptibility to calpain and enhances their binding to spectrin and PSD-95. $J$ Neurochem 79, 382-390 (2001)

53. Hai-Yan Wu, Fu-Chun Hsu, Amy J. Gleichman, Isabelle Baconguis, Douglas A. Coulter, David R. Lynch: Fyn-mediated phosphorylation of NR2B Tyr-1336 controls calpain-mediated NR2B cleavage in neurons and heterologous systems. J Biol Chem 282, 20075-20087 (2007)

54. Elisa A. Waxman, David R. Lynch: N-methyl-Daspartate receptor subtype mediated bidirectional control of p38 mitogen-activated protein kinase. $J$ Biol Chem 280, 29322-29333 (2005)

55. Chihiro Hisatsune, Hisashi Umemori, Masayoshi Mishina, Tadashi Yamamoto: Phosphorylationdependent interaction of the N-methyl-D-aspartate receptor E2 subunit with phosphatidylinositol 3-kinase. Genes Cells 4, 657-666 (1999)

56. Rachel Jurd, Claire Thornton, Jun Wang, Ken Luong, Khanhky Phamluong, Viktor Kharazia, Stuart L. Gibb, Dorit Ron: Mind bomb-2 is an E3 ligase that ubiquitinates the N-methyl-D-aspartate receptor NR2B subunit in a phosphorylation-dependent manner. $J$ Biol Chem 283, 301-310 (2008) 
57. Dorit Ron: Signaling cascades regulating NMDA receptor sensitivity to ethanol. Neuroscientist 10, 325-336 (2004)

58. Barbara Viviani, Fabrizio Gardoni, Stefano Bartesaghi, Emanuela Corsini, Alessandra Facchi, Corrado L. Galli, Monica Di Luca, Marina Marinovich: Interleukin-1B released by gp120 drives neural death through tyrosine phosphorylation and trafficking of NMDA receptors. $J$ Biol Chem 281, 30212-30222 (2006)

59. Shuo Zhang, Lambert Edelmann, Junie Liu, James E. Crandall, Maria A. Morabito: Cdk5 regulates the phosphorylation of tyrosine 1472 NR2B and the surface expression of NMDA receptors. J Neurosci 28, 415-424 (2008)

60. Graham L. Collingridge, Robin A. Lester: Excitatory amino acid receptors in the vertebrate central nervous system. Pharmacol Rev 41, 143-210 (1989)

61. Simon R. Platt: The role of glutamate in central nervous system health and disease - a review. Vet $J 173,278-86$ (2007)

62. Insuk Song, Richard L. Huganir: Regulation of AMPA receptors during synaptic plasticity. Trends Neurosci 25, 578-588 (2002)

63. Eunice Y. Yuen, Zhenglin Gu, Zhen Yan: Calpain regulation of AMPA receptor channels in cortical pyramidal neurons. $J$ Physiol 580, 241-254 (2007)

64. Ruifen Bi, Xiaoning Bi, Michel Baudry: Phosphorylation regulates calpain-mediated truncation of glutamate ionotropic receptors. Brain Res 797, 154-158 (1998)

65. Eunice E. Yuen, Wenhua Liu, Zhen Yan: The phosphorylation state of GluR1 subunits determines the susceptibility of AMPA receptors to calpain cleavage. $J$ Biol Chem 282, 16434-16440 (2007)

66. Jorge Ure, Michel Baudry, Monica Perassolo: Metabotropic glutamate receptors and epilepsy. J Neurol Sci 247, 1-9 (2006)

67. Wei Xu, Tak Pan Wong, Nadege Chery, Tara Gaertner, $\mathrm{Yu}$ Tian Wang, Michel Baudry: Calpain-mediated mGluR1-alpha truncation: a key step in excitotoxicity. Neuron 53, 399-412 (2007)

68. Kentaro Abe, Masatoshi Takeichi: NMDA-receptor activation induces calpain-mediated beta-catenin cleavages for triggering gene expression. Neuron 53, 387-397 (2007)

69. Guodong Cao, Juan Xing, Xiao Xiao, Anthony K. F. Liou, Yanqin Gao, Xiao-Ming Yin, Robert S. B. Clark, Steven H. Graham, Jun Chen: Critical role of calpain I in mitochondrial release of apoptosis-inducing factor in ischemic neuronal injury. J Neurosci 27, 9278-9293 (2007)
70. Christa L. Cortesio, Keefe T. Chan, Benjamin J. Perrin, Nicholas O. Burton, Sheng Zhang, Zhong-Yin Zhang, Anna Huttenlocher: Calpain 2 and PTP1B function in a novel pathway with Src to regulate invadopodia dynamics and breast cancer cell invasion. J Cell Biol 180, 957-971 (2008)

Abbreviations: NMDA: N-methyl-D-aspartate; AMPA: alpha-amino-3-hydroxy-5-methylisoxazole-4-propionate; mGluR: metabotropic glutamate receptor; MAP: mitogen activated protein; GFP: green fluorescent protein; PSD-95: postsynaptic density-95; SAP102: synapse associated protein 102; SAP97: synapse associated protetin 97

Key Words: Calpain, Glutamate, NMDA, AMPA, Excitotoxicity, Phosphorylation, Review

Send correspondence to: David Lynch, Division of Neurology, 502 Abramson Building, Children's Hospital of Philadelphia, $34^{\text {th }}$ Street and Civic Center Boulevard, Philadelphia, PA 19104, Tel: 215-590-2242, Fax: 215590-3779, E-mail: lynch@pharm.med.upenn.edu

http://www.bioscience.org/current/vol1S.htm 